\title{
Qualidade de cultivares de gérbera de vaso em função das características físicas e químicas dos substratos ${ }^{(1)}$
}

\author{
FERNANDA LUDWIG(2); AMARALINA CELOTO GUERRERO(2); DIRCEU MAXIMINO FERNANDES(2); \\ ROBERTO LYRA VILLAS BÔAS ${ }^{(2)}$ E DENISE LASCHI(3)
}

\begin{abstract}
RESUMO
A gérbera é uma planta que vem ganhando destaque no mercado de flores e plantas ornamentais. Com o objetivo de avaliar a preferência do consumidor quanto a cultivares de gérbera produzidas em substratos, com diferentes características físicas e químicas, realizou-se o experimento sob ambiente protegido, de setembro a novembro de 2008, na FCA/ Unesp, Botucatu (SP). O delineamento experimental foi o de blocos casualizados em esquema fatorial $5 \times 2$ (5 substratos e 2 cultivares) e quatro repetições. Os substratos utilizados foram: $1=40 \%$ terra vermelha, $40 \%$ casca de pinus, $10 \%$ composição $1\left(\mathrm{C}_{1}\right)(40 \%$ casca de pinus $+30 \%$ vermiculita $+30 \%$ casca de arroz carbonizada), $10 \%$ composição $2\left(\mathrm{C}_{2}\right)\left(75 \%\right.$ casca de pinus $+25 \%$ acícula de pinus); $2=20 \%$ terra vermelha, $30 \%$ casca pinus, $30 \% \mathrm{C}_{1}, 20 \% \mathrm{C}_{2}$; $3=50 \%$ casca de pinus, $20 \%$ fibra de coco granulada, $30 \% \mathrm{C}_{1} ; 4=30 \%$ casca de pinus, $30 \% \mathrm{C}$, $20 \%$ fibra de coco granulada, $20 \%$ fibra de coco mista; e $5=$ substrato comercial ( $70 \%$ casca de pinus $+15 \%$ turfa $+15 \%$ vermiculita). As cultivares utilizadas foram Red e Cherry. As plantas, em ponto de comercialização, foram avaliadas por consumidores com o preenchimento de questionário, atribuindo notas para o aspecto das folhas, das inflorescências e geral da planta. Em seguida, foram determinados o número e o diâmetro das inflorescências, número de folhas, diâmetro da planta, diâmetro da haste, altura da planta, área foliar, fitomassa fresca e seca e conteúdo de água da parte aérea. As notas superiores foram atribuídas à cultivar Cherry, que apresentou maior diâmetro da inflorescência e planta e menor número das inflorescências. As plantas conduzidas nos substratos com menor $\mathrm{pH}$ e maiores densidades tiveram as notas inferiores, tendo apresentado menor número de folhas, diâmetro da planta e área foliar. Conclui-se que a cultivar Cherry tem maior aceitação pelo consumidor em relação à Red e que substratos com densidade seca inferior a $530 \mathrm{~kg} \mathrm{~m}^{-3}$ e valores de $\mathrm{pH}$ entre 5,5 e 6,8 são mais adequados para produção de gérbera.
\end{abstract}

Palavras-chave: Gerbera jamesonii, cultivo sem solo, fertirrigação.

\section{ABSTRACT \\ Quality of potted gerbera cultivars as a function of physical and chemical characteristics of the substrates}

\begin{abstract}
Gerbera is a plant that has gained prominence in the market of flower and ornamental plant. Aimed at evaluating the consumer preference of gerbera cultivars produced in substrates with different physical and chemical characteristics, an experiment was carried out in greenhouse from September to November 2008 at FCA/UNESP, Botucatu (SP), Brazil. Experimental design was in randomized blocks, in factorial arrangement $5 \times 2$ ( 5 substrates and 2 cultivars), with four replicates. The substrates used were: $1=40 \%$ red soil, $40 \%$ pine bark, $10 \%$ composition 1 (C1) (40\% pine bark $+30 \%$ vermiculite and $30 \%$ carbonized rice husk), $10 \%$ composition 2 (C2) (75\% pine bark $+25 \%$ needles of pine); $2=20 \%$ red soil, $30 \%$ pine bark, $30 \% \mathrm{C} 1,20 \% \mathrm{C} 2 ; 3=50 \%$ pine bark, $20 \%$ coconut granulated fiber, $30 \% \mathrm{C} 1,4=30 \%$ pine bark, $30 \%$ $\mathrm{C} 1,20 \%$ coconut granulated fiber, $20 \%$ coconut mixed fiber; 5$)$ commercial substrate $(70 \%$ pine bark $+15 \%$ peat $+15 \%$ vermiculite) and the cultivars were Red and Cherry. Plants at the commercialization point were evaluated by consumers, who filled in a questionnaire, grading the aspect of leaves, inflorescences and overall plants. Then, inflorescence number and diameter, leaf number, plant diameter, stem diameter, plant height, leaf area, fresh and dry phytomass, and water content were assessed. The highest grades were attributed to Cherry cultivar, whi e presented the largest inflorescence and plant diameter and the smallest inflorescence number. Plants developed in substrates with lower $\mathrm{pH}$ and higher densities received the lowest grades and presented the smallest leaf number, plant diameter and leaf area. In conclusion, plant diameter, leaf area, inflorespence diameter and plant height are good indicators in the qualitative evaluation of pot gerbera plants. Cherry cultivar has increased consumer acceptance in relation to Red, and substrates with dry density of less than $530 \mathrm{~kg} \mathrm{~m}^{-3}$ and $\mathrm{pH}$ values between 5.5 and 6.8 are most suitable for the production of gerbera.
\end{abstract}

Keywords: Gerbera jamesonii, soilless cultive, fertirrigation.

\section{INTRODUÇÃO}

A floricultura, como atividade agrícola brasileira, tem apresentado acentuados crescimentos, o que aumenta, consequentemente, a demanda por pesquisas que objetivam a melhoria da quantidade e da qualidade produtiva.

O substrato para plantas exerce grande influência na produção de flores e plantas ornamentais, em especial naquelas cultivadas em vaso, onde há restrição do volume disponível para o crescimento das raízes. A seleção do substrato deve basear-se principalmente nas características físicas para um dado recipiente e um determinado manejo e para a espécie a ser cultivada a fim de contribuir para o melhor aproveitamento de água e nutrientes, determinando a melhor qualidade do produto final (FERMINO, 2003).

A caracterização física e química dos substratos é necessária para a sua correta formulação e, também, para a recomendação e monitoramento das adubações nos

\footnotetext{
(1) Recebido para publicação em 23/02/2011 e aceito em 20/05/2011.

(2) Departamento de Recursos Naturais / Ciência do Solo, FCA/UNESP. Fazenda Experimental Lageado. Caixa Postal 237, CEP: 18610-307, Botucatu, SP, Brasil.

(3) Departamento de Produção Vegetal / Horticultura, FCA/UNESP. Fazenda Experimental Lageado. Caixa Postal 237, CEP: 18610-307, Botucatu, SP, Brasil.
} 
sistemas de cultivo protegido (ABREU et al., 2007). De acordo com resultados de trabalhos com gérbera de corte, obtidos na literatura, o substrato deve apresentar alta capacidade de retenção de água, mas ao mesmo tempo deve possuir grande quantidade de macroporos para facilitar a rápida drenagem após a irrigação (ROGERS e TJIA, 1990), com resultados positivos para a precocidade, número de inflorescências por planta e qualidade uniforme (MASCARINI, 1998). Em relação às características químicas, foram registradas informações quanto ao $\mathrm{pH}$ do substrato, que deve ser mantido entre 5,5 e 6,5 (ROGERS e TJIA, 1990), com redução na produção quando o pH está acima de 6,0 (SONNEVELD e VOOGT, 1997; SAVVAS e GIZAS, 2002). Estes resultados, entretanto, limitamse à produção de gérbera de corte, sendo importante o estudo das características dos substratos para a produção de gérbera envasada, pela importância que a cultura vem obtendo no mercado de flores e plantas ornamentais.

As espécies selvagens e as cultivares de gérbera desenvolvidas inicialmente foram mais bem adaptadas para flor de corte devido a suas hastes longas. A primeira cultivar selecionada para desenvolvimento em vaso foi introduzida no Japão, no início de 1980, representando importante avanço no desenvolvimento da cultura (ROGERS e TJIA, 1990).

A qualidade de uma planta ornamental está intrinsecamenterelacionadaà subjetividade eprincipalmente à percepção individual, o que pode dificultar a classificação das plantas com maior ou menor qualidade. De modo geral, o critério de qualidade atribuído a plantas envasadas relaciona-se diretamente à composição das folhas e flores, embora não sejam reconhecidas detalhadamente as características morfológicas consideradas mais importante do ponto de vista estético.

A interação entre o potencial genético e as condições de crescimento determina a qualidade de uma planta e esses fatores devem ser orientados para satisfazer as exigências do consumidor (NOORDEGRAAF, 1994).

Desse modo, o presente trabalho foi conduzido com o objetivo de avaliar a preferência do consumidor em relação a cultivares de gérbera produzidas em substratos com diferentes características físicas e químicas.

\section{MATERIAL E MÉTODOS}

O experimento foi conduzido em ambiente protegido, de setembro a novembro de 2008, no Departamento de Recursos Naturais - Ciência do Solo, FCA - Unesp, no município de Botucatu (22 $51^{\prime}$ 'S e $48^{\circ} 26^{\prime} \mathrm{W}$ ), estado de São Paulo. A casa de vegetação utilizada apresenta cobertura em arco com plástico transparente de 150 micrômetros, com laterais de tela branca $(30 \%)$, cortinas de plástico transparente, abertas no período da manhã e fechadas à tarde, bem como em períodos chuvosos, pavimentada com concreto, com área total de $168 \mathrm{~m}^{2}$ (7 x 24 m) e 2,6 m de pé direito. A temperatura média no interior do ambiente foi de $23^{\circ} \mathrm{C}$ e a umidade relativa média do ar de $63 \%$.

O delineamento experimental utilizado foi o de blocos ao acaso, empregando o esquema fatorial $5 \times 2$ ( 5 substratos e 2 cultivares) e quatro repetições. Os substratos utilizados foram: $1=40 \%$ terra vermelha (Latossolo Vermelho
Escuro), $40 \%$ casca pinus, $10 \%$ composição $1\left(\mathrm{C}_{1}\right)(40 \%$ casca de pinus $+30 \%$ vermiculita $+30 \%$ casca de arroz carbonizada), $10 \%$ composição $2\left(\mathrm{C}_{2}\right)(75 \%$ casca de pinus $+25 \%$ acícula de pinus); $2=20 \%$ terra vermelha, $30 \%$ casca de pinus, $30 \% \mathrm{C}_{1}, 20 \% \mathrm{C}_{2} ; 3=50 \%$ casca de pinus, $20 \%$ fibra de coco granulada, $30 \% \mathrm{C}_{1} ; 4=30 \%$ casca de pinus, $30 \% \mathrm{C}_{1}, 20 \%$ fibra de coco granulada, $20 \%$ fibra de coco mista; e $5=$ substrato comercial $(70 \%$ casca de pinus $+15 \%$ turfa $+15 \%$ vermiculita). Os substratos foram selecionados a partir de análises químicas e físicas realizadas em período anterior ao experimento.

Os substratos foram caracterizados inicialmente quanto às propriedades químicas e físicas. Entre as químicas, foram determinados a condutividade elétrica (CE) e o $\mathrm{pH}$, pelo método de diluição 1:5 (BRASIL, 2007), os macronutrientes (N-NO3-, N-NH4+, K, Ca e Mg) e micronutrientes $(\mathrm{Cu}, \mathrm{Fe}, \mathrm{Mn}$ e $\mathrm{Zn})$, quantificados pelo método de diluição do 1:1,5 (SONNEVELD e ELDEREN, 1994). Para o pH, determinaram-se também os valores ao final do experimento. Entre as físicas, foram determinados a densidade úmida e seca (BRASIL, 2007), a curva de retenção de água, a porosidade total, os sólidos (DE BOODT e VERDONCK,' 1972) e a granulometria (MARTINEZ, 1992). Os valores são apresentados na Tabela 1 .

Foram utilizadas mudas de gérbera (Gerbera jamesonii L.) com quatro folhas definitivas, cultivares Cherry e Red, pertencentes a série "Dark eyes" da empresa Sakata ${ }^{\circledR}$. As mudas foram transplantadas em vasos com capacidade para $1 \mathrm{~L}$ (11,5 cm de altura, $13 \mathrm{~cm}$ de base superior e $9 \mathrm{~cm}$ de base inferior), preenchidos com o substrato correspondente ao tratamento, de acordo com a densidade úmida.

As plantas foram aclimatadas durante 30 dias, dispostas sobre bancada de madeira (1,2 $\mathrm{m}$ de largura, $12 \mathrm{~m}$ de comprimento e $0,8 \mathrm{~m}$ de altura) sob malha termorrefletora (Aluminet ${ }^{\circledR}$ ) que mantinha a intensidade luminosa próxima a 25.000 Lux. Após a aclimatação, os vasos foram espaçados de 25 em 25 $\mathrm{cm}$ e distribuídos em duas bancadas, permanecendo na intensidade luminosa máxima de 50.000 Lux, com o uso de malha termorrefletora, disposta na parte superior interna da casa de vegetação.

O manejo da fertirrigação baseou-se na pesagem diária dos vasos. A lâmina necessária para a reposição de água foi estabelecida pela diferença entre a massa obtida na capacidade de vaso (100\% de água disponível) e a capacidade mínima de retenção, ou ponto de murcha permanente. A partir dessas medidas, aferiu-se a massa correspondente a 50 e $25 \%$ da água disponível no vaso.

A solução nutritiva apresentou a seguinte composição em mg L ${ }^{-1}: 121 \quad \mathrm{~N}^{-\mathrm{NO}_{3}}{ }^{-}$, $12{\mathrm{~N}-\mathrm{NH}_{4}}^{+}, 92 \mathrm{~K}, 24 \mathrm{P}, 175 \mathrm{Ca}, 27 \mathrm{Mge} 39 \mathrm{~S}, 0,19 \mathrm{~B}, 0,08 \mathrm{Cu}$, 2,74 Fe, 0,19 Mn, 0,04 Mo e 0,08 Zn no período vegetativo; e $168 \mathrm{~N}^{-\mathrm{NO}_{3}}{ }^{-}, 41 \mathrm{~N}^{-\mathrm{NH}_{4}}{ }^{+}, 303 \mathrm{~K}, 35 \mathrm{P}, 105 \mathrm{Ca}, 45 \mathrm{Mg}$ e $55 \mathrm{~S}, 0,19 \mathrm{~B}, 0,08 \mathrm{Cu}, 2,74 \mathrm{Fe}, 0,19 \mathrm{Mn}, 0,04 \mathrm{Mo}$ e $0,08 \mathrm{Zn}$ no período reprodutivo.

$\mathrm{O}$ volume total de solução nutritiva aplicada por planta foi de 3,9 L no substrato 1; 3,2 L no substrato 2; 4,2 L no substrato 3; 4,7 L no substrato 4; e 4,1 L no substrato 5. Foi feito o manejo da CE da solução nutritiva duas vezes por semana a fim de manter em $2,0 \mathrm{dS} \mathrm{m}^{-1}$ no período vegetativo e $2,5 \mathrm{dS} \mathrm{m}^{-1}$ no 
período reprodutivo.

As plantas em ponto de comercialização (LIN e FRENCH, 1985) foram expostas em lugar público, na Faculdade de Ciências Agronômicas de Botucatu, SP, passíveis de análise por transeuntes (considerados potenciais consumidores). Quarenta e cinco pessoas receberam um formulário com o objetivo de avaliar o aspecto das folhas, das flores e as condições gerais da planta, indicando ainda sua intenção de compra. A escala de notas utilizada foi de: (1) ruim, (2) bom, (3) muito bom e (4) excelente. Para essa avaliação, o delineamento utilizado foi o inteiramente casualizado, em esquema fatorial $5 \times 2$ (5 substratos e 2 cultivares) e 45 repetições, representando o número de entrevistados.

Em seguida, foram determinados o número de folhas por planta, o diâmetro da planta $(\mathrm{cm})$, a altura da planta (cm), o diâmetro das inflorescências ( $\mathrm{mm})$ e da haste $(\mathrm{mm})$, no momento anterior ao corte das plantas. Após o corte da parte aérea, foram aferidas a área foliar $\left(\mathrm{dm}^{2}\right)$, a fitomassa fresca e seca $(\mathrm{g})$ das folhas, inflorescências e total, tendo sido calculado o conteúdo total da água na parte aérea $(\mathrm{g})$.

O diâmetro de planta foi medido com uso de régua graduada a partir de dois pontos extremos e perpendiculares entre si. A altura da planta foi determinada com uso de régua graduada a partir da porção superior do vaso até a extremidade superior da planta. O diâmetro das inflorescências foi aferido com uso de paquímetro digital a partir de dois pontos extremos e perpendiculares entre si. O diâmetro de haste foi determinado $5 \mathrm{~cm}$ abaixo da inserção da inflorescência, com uso de paquímetro digital. A área foliar foi determinada em medidor modelo $\mathrm{Li} 3100$, da marca Licor. A fitomassa fresca da parte aérea das plantas foi aferida em balança digital, sendo em seguida colocada em estufa de ventilação forçada a $65^{\circ} \mathrm{C}$ por um período de 48 horas, obtendo-se a fitomassa seca em balança digital.

Os resultados foram submetidos à análise de variância pelo teste $\mathrm{F}$ e as médias comparadas pelo teste Tukey a $5 \%$ de probabilidade, realizando-se a transformação das notas para a função logaritmo $(\log (\mathrm{X}))$. Procedeu-se ao desdobramento da interação entre substratos e cultivares quando a análise de variância revelou efeito significativo, utilizando o programa estatístico Sisvar (FERREIRA, 2000). Determinou-se, também, a correlação linear entre as características avaliadas.

\section{RESULTADOS E DISCUSSÃO}

A cultivar Cherry obteve maiores notas quanto ao aspecto das folhas em relação à Red quando conduzida nos substratos 1, 4 e 5, não diferindo significativamente nos demais substratos (Tabela 2). $\mathrm{O}$ aspecto das folhas recebeu notas diferenciadas quando a cultivar Cherry foi conduzida nos diferentes substratos, sendo as maiores atribuídas àquelas desenvolvidas nos substratos 4 e 5 , que diferiram significativamente do substrato 2 .

As menores notas atribuídas às plantas conduzidas no substrato 2 estão relacionadas principalmente ao número de folhas, diâmetro de planta e área foliar, que foram inferiores (Tabela 3). Isso indica que as folhas das plantas conduzidas nesse substrato foram menos desenvolvidas, havendo maior preferência, pelo consumidor, por vasos com boa estrutura de folhas, especialmente com elevada área foliar.

O diâmetro de planta e área foliar foi superior para Cherry (Tabela 3), indicando também que essas características são importantes para a comercialização da gérbera de vaso, ou seja, o diâmetro de planta de 29 a $31 \mathrm{~cm}$ e área foliar superior a $10 \mathrm{dm}^{2}$ são características que conferem à planta maior probabilidade de ser comercializada, em relação àquelas com valores inferiores. A correlação dessas variáveis corrobora as notas atribuídas às plantas, pois o diâmetro de planta apresentou $\mathrm{r}$ de $0,77^{* *}$ e área foliar de $0,81 * *$, ambos referentes às notas atribuídas às folhas. Para o número de folhas, a correlação não foi significativa $\left(\mathrm{r}: 0,34^{\mathrm{NS}}\right.$ ). característica que mais se correlacionou com a área foliar foi o diâmetro de planta (r: $\left.0,87^{* *}\right)$, seguido pelo número de folhas (r: $\left.0,67^{* *}\right)$.

A área foliar das plantas foi influenciada pelas características genéticas da cultivar, concordando com LUDWIG (2007) e MOTA(2007). Obtevese uma área foliar significativamente inferior para a cultivar Red, com 9,99 $\mathrm{dm}^{2}$ em relação à Cherry, com 11,08 $\mathrm{dm}^{2}$. GUERRERO (2009), ao estudar o efeito da aplicação do potássio na cultivar Red, obteve área foliar média de $8 \mathrm{dm}^{2}$, indicando que esta cultivar é de menor porte.

A fitomassa fresca de folha não variou significativamente entre as cultivares, mas sim quando essas foram desenvolvidas nos diferentes substratos, sendo superior nos substratos 3, 4 e 5, plantas essas que apresentaram maior conteúdo de água na parte aérea (Tabela 3). Entretanto, a fitomassa seca foi superior para Cherry, bem como nos substratos 3,4 e 5 . A correlação da fitomassa fresca e seca de folha e notas atribuídas às folhas também foi significativa $(0,77 * *$ e $0,89 * *$, respectivamente). Esses dados contribuem para a afirmação de que a formação de folhas é um indicativo de qualidade para plantas de gérbera de vaso.

O efeito da interação entre substratos e cultivares foi verificado para a fitomassa fresca e seca total (Tabelas 3 e 4). A cultivar Cherry apresentou maior fitomassa fresca quando conduzida no substrato 2 e seca quando conduzida nos substratos 1 e 2 , em relação à Red. Os menores valores para a fitomassa fresca e seca, para as duas cultivares, ocorreram nos substratos 1 e 2, o que pode estar relacionado com as características de baixo $\mathrm{pH}$ e alta $\mathrm{CE}$, além da menor porosidade total e água disponível, relacionada principalmente com a reduzida porcentagem de partículas inferiores a $5 \mathrm{~mm}$, responsáveis pela microporosidade e retenção de água (Tabela 1$)$.

As notas conferidas às inflorescências foram dependentes dos substratos e cultivares, tendo sido superiores para a cultivar Cherry, nos substratos 1 e 5, e inferiores no substrato 3, quando comparada com a cultivar Red (Tabela 2). Analisando os substratos em cada cultivar de forma isolada, foi possível observar que Red apresentou maiores notas nos substratos 3, 4 e 5 e Cherry no substrato 5 , sem diferir significativamente nos substratos 1 e 4 . O número de inflorescência parece não ter sido decisivo para a atribuição das notas (r: $-0,59^{\mathrm{ns}}$ ), e sim o diâmetro de inflorescência (r: 0,87**). Esses dados apresentaram correlação negativa entre si 
(r: $\left.-0,88^{* *}\right)$, indicando que quanto maior o número das inflorescências emitidas, menor é o seu diâmetro.

Segundo NOORDEGRAAF (1994), os parâmetros de qualidade dependem do tipo de cultura, e no caso de plantas floríferas, o número de flores pode ser considerado um indicativo de qualidade. Entretanto, essa característica foi menos relevante do que o diâmetro da inflorescência da gérbera, já que o mínimo necessário de inflorescências exigido pelo mercado, de duas inflorescências abertas, foi atingido nas duas cultivares, visto que as médias foram de 3,15 para Cherry e 4,70 para Red.

A cultivar Red caracterizou-se por ser uma cultivar mais florífera em relação à Cherry, que apresentou menos inflorescências, porém de maior tamanho, características estas devido ao potencial genético. Verificou-se uma tendência de melhor aceitação por plantas constituídas por inflorescências com maior diâmetro, mesmo que seu número seja inferior.

O diâmetro da haste foi maior para a cultivar Cherry quando conduzida nos substratos 1, 2 e 4 (Tabela 4). Correlação linear positiva e altamente significativa (r: $0,89 * *$ ) foi constatada entre o diâmetro da haste e da inflorescência. A importância dessa informação está relacionada principalmente à sustentação das inflorescências, evitando o possível tombamento e perda de qualidade, principalmente durante transporte e manuseio.

A qualidade das plantas envasadas baseia-se também na altura delas e considera principalmente que, para uma boa conformação de vaso, a planta apresente 1,5 a 2 vezes a altura do vaso (GUERRERO, 2009). Portanto, todas as plantas se enquadr $\mathrm{e}_{\mathrm{a}}$ nesse padrão qualitativo.

A altura da planta não foi significativamente diferente entre as cultivares de gérbera. Para a cultivar Cherry, LUDWIG (2007) registrou valores superiores (29,7 $\mathrm{cm}$ ), constatando diferença entre cultivares para esta característica. As diferenças em relação à altura estão principalmente relacionadas ao ciclo de produção, que foi de 60 dias, para o trabalho citado. GUERRERO (2009) obteve altura média de planta de $20 \mathrm{~cm}$ ao conduzir plantas de gérbera da cultivar Red.

As notas atribuídas às plantas e à altura relacionaramse positivamente (r: $\left.0,73^{*}\right)$, podendo ser um indicativo de que alturas de plantas inferiores a $18 \mathrm{~cm}$ são menos adequadas que alturas próximas a $23 \mathrm{~cm}$.

A qualidade externa de uma cultura ornamental está relacionada diretamente com a cultivar que apresenta um maior ou menor potencial genético, que pode ser expresso mais facilmente quando os fatores de produção são adequados, entre eles, o substrato. De acordo com NOORDEGRAAF (1994), a qualidade de uma planta é determinada pelo potencial genético e pelas condições de crescimento.

As notas atribuídas ao aspecto geral da planta foram superiores estatisticamente para Cherry em relação à Red quando desenvolvida nos substratos 1 e 4 . Maiores notas para as duas cultivares foram registradas nos substratos 3, 4 e 5 (Tabela 2), substratos estes que apresentaram menor densidade úmida e seca, maior porosidade total e água disponível, reflexo de uma distribuição uniforme de partículas com diferentes tamanhos (Tabela 1). Essas características físicas podem influenciar positivamente no desenvolvimento e qualidade das plantas.

Os parâmetros de qualidade variam entre os tipos de planta ornamental. Especificamente para flor envasada, há que se considerar não somente a inflorescência, mas também a folhagem, e mais importante, o conjunto formado pelas inflorescências e folhas.

Verificou-se que 75 e $54 \%$ dos entrevistados não comprariam as cultivares Red e Cherry, respectivamente, se conduzidas no substrato 2 , indicando elevada rejeição das plantas desenvolvidas neste substrato. Já Cherry, no substrato 4, teve aceitação de 94\%, demonstrando que a cultivar, ao ser conduzida neste substrato, apresenta qualidade adequada para comercialização (Figura 1).

Os resultados obtidos indicam que a qualidade final de plantas de gérbera de vaso relaciona-se com o substrato utilizado, principalmente pelas características físicas e químicas. Entre estas características, destacamse as físicas, que devem ser as ideais no momento da seleção do substrato, já que sua modificação durante o desenvolvimento da cultura é dificultada.

A qualidade de uma planta é influenciada pela água fornecida pela irrigação, e o fornecimento da água depende diretamente do substrato utilizado. Assim, um substrato ideal apresenta a característica de fornecer água para a planta sem comprometer a oxigenação no ambiente radicular (NOORDEGRAAF, 1994). Com base nessa informação e nos resultados obtidos, pode-se inferir que substratos com espaço de aeração de $17 \%$ e água disponível de $36 \%$ são adequados para a produção de plantas de gérbera, refletindo em melhor qualidade.

Embora o espaço de aeração nos substratos 1 e 2 esteja adequado, segundo recomendação de DE BOODT e VERDONCK (1972), a densidade seca apresentou-se elevada (Tabela 1), devido principalmente ao uso de solo com características argilosas e maior possibilidade de compactação, o que pode ter afetado a oxigenação no ambiente radicular e, consequentemente, a redução no desenvolvimento da planta. KÄMPF (2000) recomenda utilizar substratos com densidade seca de 250 a $400 \mathrm{~kg} \mathrm{~m}^{-3}$ para vasos de até $15 \mathrm{~cm}$ de altura, afirmando que altas densidades limitam o crescimento das plantas.

Vários autores sugerem que o valor mínimo de $\mathrm{pH}$ do substrato para a cultura da gérbera seja de 5,5 (CAVINS et al., 2000; ROGERS e TJIA, 1990), justificando os resultados obtidos em que as plantas com as características morfológicas inferiores foram registradas nos substratos 1 e 2, com baixos índices de $\mathrm{pH}$ (Tabela 1). Plantas cultivadas em ambientes ácidos têm quantidades menores de nutrientes à sua disposição, além de ficarem sujeitas à maior absorção de elementos tóxicos como Al e Mn (FERMINO, 1996). Os maiores teores de $\mathrm{Mn}$ foram registrados no substrato 2 (Tabela 1), o que também se refletiu em maior teor na planta (dados não apresentados), com valores médios de $650 \mathrm{mg} \mathrm{kg}^{-1}$, e menores no substrato 5, com valores médios de $49 \mathrm{mg} \mathrm{kg}^{-1}$, concordando com o autor.

\section{CONCLUSÃO}

Conclui-se que a cultivar Cherry tem maior aceitação pelo consumidor em relação à Red e que substratos com 
densidade seca inferior a $530 \mathrm{~kg} \mathrm{~m}^{-3}$ e valores de $\mathrm{pH}$ entre 5,5 e 6,8 são mais adequados para produção de gérbera.

\section{REFERÊNCIAS}

ABREU, M.F.;ABREU, C.A.; SARZI, I.; PADUAJUNIOR, A.L. Extratores aquosos para a caracterização química de substratos para plantas. Horticultura Brasileira, Brasília, v. 25, n. 2, p. 184-187. 2007.

BRASIL. Instrução Normativa n.17, de 21 de maio de 2007. Aprova os Métodos Analíticos Oficiais para Análise de Substratos e Condicionadores de Solos. Diário Oficial da União, Brasília, 24 maio. 2007. Seção 1, p.8.

CAVINS, T.J.; FONTENO, W.C.; HARDEN, B.; McCALL, I.; GIBSON, J.L. Monitoring and managing $\mathbf{p H}$ and EC using the PourThru extraction method. Raleigh: Horticulture Information, 2000. 17p.

DE BOODT, M.; VERDONCK, O. The physical properties of the substrates in horticulture. Acta Horticulturae, Wageningen, v.1, n.23, p.37-44, 1972.

FERMINO, M.H. Aproveitamento de resíduos industriais e agrícolas como alternativas de substratos hortícolas. Porto Alegre, UFRGS, 1996. 90p. Tese (Mestrado em Fitotecnia).

FERMINO, M.H. Métodos de análise para caracterização física de substratos para planta. Porto Alegre, UFRGS, 2003. 89p. Tese (Doutorado em Fitotecnia).

FERREIRA, D.F. Análises estatísticas por meio do Sisvar para Windows versão 4.0. In: REUNIÃO ANUAL DA REGIÃO BRASILEIRA DA SOCIEDADE INTERNACIONAL DE BIOMETRIA, 45, São Carlos, 2000. Anais...UFSCar, São Carlos, 2000. p.255-258.

GUERRERO, A.C. Aplicação de cloreto e silicato de potássio em gérbera (Gerbera jamesonii L.) de vaso. Botucatu: Faculdade de Ciências Agronômicas, UNESP, 2009. 80p. Dissertação (Mestrado em Agronomia/ Horticultura).

KÄMPF, A.N. Produção comercial de plantas ornamentais. (Ed), Guaíba, 2000. 254p.
LIN, W.C.; FRENCH, C.J. Effects of supplementary lighting and soil warming on flowering of three gerbera cultivars. HortScience, Alexandria, v.20, n.2, p.271-273, 1985.

LUDWIG, F. Cultivares de gérbera (Gerbera jamesonii L.), em vaso, sob dois níveis de fertirrigação. Botucatu: Faculdade de Ciências Agronômicas, UNESP, 2007. 79p. Dissertação (Mestrado em Agronomia/Horticultura).

MARTINEZ, F.X. Propuesta de metodologia para la determinacion de lãs propriedades físicas de los substratos. Actas de las I jornadas de substratos de la SECH, Madrid, v. 294, p. 55-66, 1992.

MASCARINI, L. Gerbera cultivation in growing media. Horticultura Internacional, Tarragona, v. 6, n. 19, p. 86$88,1998$.

MOTA, P.R.D. Aplicação via fertirrigação de soluções com diferentes condutividades elétricas para produção de gérbera (Gerbera jamesonii L.) sob ambiente protegido. Botucatu: Faculdade de Ciências Agronômicas, UNESP, 2007. 149p. Tese (Doutorado em Agronomia/ Irrigação e Drenagem.

NOORDEGRAAF, C.V. Production and marketing of high quality plants. Acta Horticulturae, Wageningen, v.353, p.134-147, 1994.

ROGERS, M.N., TJIA, B.O. Gerbera production. Timber Press Growers handbook series, Portland, Oregon. v.4, 1990. 116p.

SAVVAS, D.; GIZAS, G. Response of hydroponically grown gerbera to nutrient solution recycling and different cation ratios. Scientia Horticulturae, Amsterdam, v. 96, p. 267-280, 2002.

SONNEVELD, C.; ELDEREN, C.W. Chemical analysis of peat growing media by means of water extraction. Communication on Soil Science and Plant Analysis, USA, v. 25, p. 3199-3208, 1994.

SONNEVELD, C.; VOOGT, W. Effects of $\mathrm{pH}$ and Mn application on yield and nutrient absorption with rockwool grown gerbera (refereed). Acta Horticulturae, Wageningen, v. 450, p. 139-147, 1997. 
Tabela 1. Características químicas e físicas dos substratos utilizados no experimento. Botucatu (SP), 2008

Table 1. Chemical and physical characteristics of substrates used in the experiment. Botucatu (SP), 2008

\begin{tabular}{|c|c|c|c|c|c|}
\hline Características & $\begin{array}{c}\text { Substrato } \\
1 \\
\end{array}$ & $\begin{array}{l}\text { Substrato } \\
2 \\
\end{array}$ & $\begin{array}{c}\text { Substrato } \\
3 \\
\end{array}$ & $\begin{array}{c}\text { Substrato } \\
4 \\
\end{array}$ & $\begin{array}{c}\text { Substrato } \\
5 \\
\end{array}$ \\
\hline \multicolumn{6}{|l|}{ Químicas } \\
\hline $\mathrm{CE}_{1: 5}\left(\mathrm{dS} \mathrm{m}^{-1}\right)$ & 0,54 & 0,49 & 0,26 & 0,28 & 0,48 \\
\hline $\mathrm{pH}_{1: 5 \text { (inicial) }}$ & 5,13 & 4,81 & 5,74 & 5,74 & 7,58 \\
\hline $\mathrm{pH}_{1: 5 \text { (final) }}$ & 5,42 & 4,86 & 5,63 & 5,59 & 6,89 \\
\hline $\mathrm{N}^{-\mathrm{NH}_{4}}{ }_{1: 1,5}\left(\mathrm{mg} \mathrm{L}^{-1}\right)$ & 1,99 & 3,98 & 1,41 & 1,75 & 1,56 \\
\hline $\mathrm{N}^{-\mathrm{NO}_{3}}{ }_{1: 1,5}^{-}\left(\mathrm{mg} \mathrm{L}^{-1}\right)$ & 77,99 & 122,17 & 2,51 & 12,72 & 7,10 \\
\hline $\mathrm{K}_{1: 1,5}\left(\mathrm{mg} \mathrm{L}^{-1}\right)$ & 70,25 & 86,00 & 45,43 & 56,70 & 176,25 \\
\hline $\mathrm{Ca}_{1: 1,5}\left(\mathrm{mg} \mathrm{L}^{-1}\right)$ & 52,40 & 84,98 & 3,57 & 22,83 & 21,63 \\
\hline $\operatorname{Mg}_{1: 1,5}\left(\mathrm{mg} \mathrm{L}^{-1}\right)$ & 50,15 & 57,60 & 12,13 & 24,45 & 12,83 \\
\hline $\mathrm{Cu}_{1: 1,5}\left(\mathrm{mg} \mathrm{L}^{-1}\right)$ & 0,013 & 0,015 & 0,06 & 0,028 & 0,063 \\
\hline $\mathrm{Fe}_{1: 1,5}\left(\mathrm{mg} \mathrm{L}^{-1}\right)$ & 0,383 & 0,62 & 5,568 & 1,763 & 1,825 \\
\hline $\mathrm{Mn}_{1: 1,5}\left(\mathrm{mg} \mathrm{L}^{-1}\right)$ & 0,19 & 2,48 & 0,203 & 0,378 & 0,838 \\
\hline $\mathrm{Zn}_{1: 1,5}\left(\mathrm{mg} \mathrm{L}^{-1}\right)$ & 0,038 & 0,11 & 0,063 & 0,060 & 0,083 \\
\hline \multicolumn{6}{|l|}{ Físicas } \\
\hline PT (\%) & 78,79 & 80,62 & 84,28 & 89,18 & 83,51 \\
\hline S (\%) & 21,21 & 19,38 & 15,72 & 10,82 & 16,49 \\
\hline EA $(\%)$ & 23,50 & 19,33 & 12,76 & 17,15 & 21,10 \\
\hline $\mathrm{AD}(\%)$ & 28,74 & 29,40 & 37,34 & 35,80 & 32,90 \\
\hline AR (\%) & 26,48 & 31,89 & 34,62 & 36,23 & 29,50 \\
\hline $\mathrm{DU}\left(\mathrm{kg} \mathrm{m}^{-3}\right)$ & 750 & 850 & 690 & 590 & 530 \\
\hline $\operatorname{DS}\left(\mathrm{kg} \mathrm{m}^{-3}\right)$ & 538 & 540 & 450 & 298 & 340 \\
\hline $\mathrm{DP}>4 \mathrm{~mm}(\%)$ & 15,9 & 7,5 & 16,4 & 16,2 & 2,1 \\
\hline DP 2-4 mm (\%) & 24,0 & 21,9 & 20,3 & 18,2 & 23,5 \\
\hline DP $1-2$ mm (\%) & 20,5 & 23,6 & 17,5 & 17,3 & 20,4 \\
\hline DP $0,5-1 \mathrm{~mm}(\%)$ & 28,5 & 32,9 & 21,1 & 21,6 & 21,0 \\
\hline DP 0,25-0,5 mm (\%) & 8,6 & 11,1 & 13,3 & 14,3 & 14,8 \\
\hline $\mathrm{DP}<0,25 \mathrm{~mm}(\%)$ & 2,6 & 3,0 & 11,5 & 12,4 & 18,3 \\
\hline
\end{tabular}

Substratos: $1=40 \%$ terra vermelha (Latossolo Vermelho Escuro), 40\% casca pinus, $10 \%$ composição $1\left(C_{1}\right)(40 \%$ casca de pinus $+30 \%$ vermiculita $30 \%$ casca de arroz carbonizada), $10 \%$ composição $2\left(\mathrm{C}_{2}\right)(75 \%$ casca de pinus $+25 \%$ acícula de pinus $) ; 2=20 \%$ terra vermelha, $30 \%$ casca de pinu: $30 \% \mathrm{C}_{1}, 20 \% \mathrm{C}_{2} ; 3=50 \%$ casca de pinus, $20 \%$ fibra de coco granulada, $30 \% \mathrm{C}_{1} ; 4=30 \%$ casca de pinus, $30 \% \mathrm{C}_{1}, 20 \%$ fibra de coco granulada, $20 \%$, fibra de coco mista; e $5=$ substrato comercial $(70 \%$ casca de pinus $+15 \%$ turfa $+15 \%$ vermiculita).

CE: condutividade elétrica, PT: porosidade total, S: sólidos, EA: espaço de aeração, AD: água disponível, AR: água remanescente, DU: densidade úmida, DS: densidade seca, DP: distribuição de partículas.

CE: eletrical condutivity, PT: total porosity, S: solids, EA: aeration space, AD: available water, AR: remaining water, DU: humid bulk density, D: dry bulk density, DP: particles distribution. 
Tabela 2. Notas atribuídas ao aspecto das folhas, aspecto das inflorescências e aspecto geral da planta. Botucatu (SP). 2008 Table 2. Grades attributed to the leaves, flowers and general appearance of the plant. Botucatu (SP). 2008

\begin{tabular}{|c|c|c|c|c|c|c|}
\hline \multirow{3}{*}{ Substrato } & \multicolumn{6}{|c|}{ Notas atribuídas } \\
\hline & \multicolumn{2}{|c|}{ Folhas } & \multicolumn{2}{|c|}{ Inflorescências } & \multicolumn{2}{|r|}{ Geral } \\
\hline & Red & Cherry & Red & Cherry & Red & Cherry \\
\hline 1 & 1,71 a $\mathrm{B}$ & $2,74 \mathrm{ab} \mathrm{A}$ & $2,20 \mathrm{~b} \mathrm{~B}$ & $2,89 \mathrm{ab} \mathrm{A}$ & $1,89 \mathrm{~b} \mathrm{~B}$ & $2,61 \mathrm{~b} \mathrm{~A}$ \\
\hline 2 & 1,80 a $\mathrm{A}$ & $2,35 \mathrm{~b} \mathrm{~A}$ & $2,15 \mathrm{~b} \mathrm{~A}$ & $2,35 \mathrm{c} \mathrm{A}$ & $2,07 \mathrm{~b} \mathrm{~A}$ & $2,39 \mathrm{~b} \mathrm{~A}$ \\
\hline 3 & 2,74 a $\mathrm{A}$ & $2,74 \mathrm{ab} A$ & 3,07 a A & $2,70 \mathrm{bc} B$ & 2,91 a $A$ & $2,72 \mathrm{ab} A$ \\
\hline 4 & 2,65 a B & 3,13 a $\mathrm{A}$ & 2,91 a $\mathrm{A}$ & $3,13 \mathrm{ab} A$ & 2,67 a B & 3,15 a $\mathrm{A}$ \\
\hline 5 & 2,46 a B & 2,85 a A & 2,76 a $B$ & 3,26 a A & 2,61 a A & $2,80 \mathrm{ab} \mathrm{A}$ \\
\hline CV (\%) & \multicolumn{2}{|c|}{31,70} & \multicolumn{2}{|c|}{32,66} & \multicolumn{2}{|r|}{32,88} \\
\hline
\end{tabular}

Substratos: $1=40 \%$ terra vermelha (Latossolo Vermelho Escuro), $40 \%$ casca pinus, $10 \%$ composição $1(\mathrm{C})(40 \%$ casca de pinus $+30 \%$ vermiculita + $30 \%$ casca de arroz carbonizada), $10 \%$ composição $2\left(\mathrm{C}_{2}\right)(75 \%$ casca de pinus $+25 \%$ acícula de pinus $) ; 2=20 \%$ terra vermelha, $30 \%$ casca de pinus, $30 \% \mathrm{C}_{1}, 20 \% \mathrm{C}_{2} ; 3=50 \%$ casca de pinus, $20 \%$ fibra de coco granulada, $30 \% \mathrm{C}_{1} ; 4=30 \%$ casca de pinus, $30 \% \mathrm{C}_{1}, 20 \%$ fibra de coco granulada, $20 \%$ fibra de coco mista; e $5=$ substrato comercial ( $70 \%$ casca de pinus $+15 \%$ turfa $+15 \%$ vermiculita). Escala de notas: (1) ruim, (2) bom, (3) muito bom e (4) excelente. Médias seguidas pela mesma letra minúscula na coluna e maiúscula na linha, não diferem entre si pelo teste Tukey a 5\%.

Grades: (1) poor, (2) good, (3) very good, (4) excelent. Means followed by the same lowercase letters in the same column and uppercase letters in the same line did not differ by the Tukey test at $5 \%$.

Tabela 3. Valores médios de número das folhas (NF), diâmetro da planta (DP), área foliar (AF), fitomassa fresca das folhas (FFF), inflorescência (FFI) e total (FFT), fitomassa seca das folhas (FSF), inflorescência (FSI) e total (FST), conteúdo da água total na parte aérea (CAT) altura da planta (A), número de inflorescência (NI), diâmetro das inflorescências (DI) e diâmetro de haste (DH) em plantas de gérbera, em função dos substratos e cultivares. Botucatu (SP). 2008.

Table 3. Leaf number (NF), plant diameter $(D P)$, leaf area $(A F)$, leaffresh phytomass $(F F F)$, inflorescence fresh phytomass (FFI), total fresh phytomass (FFT), leaf dry phytomass (FSF), inflorescence dry phytomass (FSI), total dry phytomass (FST), total water content in aerial part (CAT), inflorescence number (NI) plant height (A), inflorescence diameter (DI) and stem diameter (DH) at gerbera plants, as function as substrates and cultivars. Botucatu (SP). 2008

\begin{tabular}{|c|c|c|c|c|c|c|c|c|c|c|c|c|c|c|}
\hline Substrato & $\mathrm{NF}$ & $\begin{array}{c}\text { DP } \\
--\mathrm{cm}--\end{array}$ & $\begin{array}{c}\mathrm{AF} \\
--\mathrm{dm}^{2}-\end{array}$ & $\begin{array}{l}\text { FFF } \\
-----\end{array}$ & FFI & FFT & FSF & FSI & FST & CAT & NI & $\begin{array}{c}\text { A } \\
---\mathrm{cm}--\end{array}$ & DI & DH \\
\hline 1 & $\begin{array}{c}28,63 \\
a b\end{array}$ & $\begin{array}{c}27,53 \\
b c\end{array}$ & $\begin{array}{c}8,90 \\
\text { b }\end{array}$ & $\begin{array}{c}38,76 \\
\text { b }\end{array}$ & $\begin{array}{c}31,29 \\
\mathrm{a}\end{array}$ & $\begin{array}{c}70,05 \\
\mathrm{a}\end{array}$ & $\begin{array}{c}6,13 \\
\mathrm{~b}\end{array}$ & $\begin{array}{c}4,97 \\
\mathrm{a}\end{array}$ & $\begin{array}{c}11,10 \\
\mathrm{a}\end{array}$ & $\begin{array}{c}58,94 \\
\text { b }\end{array}$ & $\begin{array}{c}4,89 \\
\mathrm{a}\end{array}$ & $\begin{array}{c}18,00 \\
b c\end{array}$ & $\begin{array}{c}71,62 \\
a\end{array}$ & $\begin{array}{c}3,68 \\
a\end{array}$ \\
\hline 2 & $\begin{array}{c}21,25 \\
\mathrm{~b}\end{array}$ & $\begin{array}{c}25,97 \\
\mathrm{c}\end{array}$ & $6,77 \mathrm{c}$ & $\begin{array}{c}30,78 \\
\text { b }\end{array}$ & $\begin{array}{c}25,53 \\
\mathrm{a}\end{array}$ & $\begin{array}{c}56,32 \\
\mathrm{a}\end{array}$ & $\begin{array}{c}5,07 \\
\mathrm{~b}\end{array}$ & $\begin{array}{c}4,13 \\
\mathrm{a}\end{array}$ & $\begin{array}{c}9,20 \\
\mathrm{a}\end{array}$ & $\begin{array}{c}47,12 \\
\mathrm{c}\end{array}$ & $\begin{array}{c}4,00 \\
\mathrm{a}\end{array}$ & $\begin{array}{c}17,75 \\
\mathrm{c}\end{array}$ & $\begin{array}{c}72,96 \\
a\end{array}$ & $\begin{array}{c}3,94 \\
\mathrm{a}\end{array}$ \\
\hline 3 & $\begin{array}{c}33,63 \\
a\end{array}$ & $\begin{array}{c}29,72 \\
\text { ab }\end{array}$ & $\begin{array}{c}12,27 \\
a\end{array}$ & $\begin{array}{c}58,20 \\
\mathrm{a}\end{array}$ & $\begin{array}{c}34,08 \\
\mathrm{a}\end{array}$ & $\begin{array}{c}92,28 \\
\text { a }\end{array}$ & $\begin{array}{c}8,49 \\
\mathrm{a}\end{array}$ & $\begin{array}{c}5,21 \\
\mathrm{a}\end{array}$ & $\begin{array}{c}13,70 \\
\mathrm{a}\end{array}$ & $\begin{array}{c}78,58 \\
\mathrm{a}\end{array}$ & $\begin{array}{c}3,63 \\
\mathrm{a}\end{array}$ & $\begin{array}{c}23,13 \\
a b c\end{array}$ & $\begin{array}{c}83,76 \\
a\end{array}$ & $\begin{array}{c}4,20 \\
\mathrm{a}\end{array}$ \\
\hline 4 & $\begin{array}{c}29,25 \\
\text { ab }\end{array}$ & $\begin{array}{c}31,44 \\
\mathrm{a}\end{array}$ & $\begin{array}{c}12,88 \\
\mathrm{a}\end{array}$ & $\begin{array}{c}61,34 \\
\mathrm{a}\end{array}$ & $\begin{array}{c}36,73 \\
\mathrm{a}\end{array}$ & $\begin{array}{c}98,07 \\
\mathrm{a}\end{array}$ & $\begin{array}{c}9,47 \\
\mathrm{a}\end{array}$ & $\begin{array}{c}5,74 \\
\mathrm{a}\end{array}$ & $\begin{array}{c}15,21 \\
\mathrm{a}\end{array}$ & $\begin{array}{c}82,86 \\
\text { a }\end{array}$ & $\begin{array}{c}3,63 \\
\mathrm{a}\end{array}$ & $\begin{array}{c}23,50 \\
a b\end{array}$ & $\begin{array}{c}85,91 \\
\mathrm{a}\end{array}$ & $\begin{array}{c}4,45 \\
\mathrm{a}\end{array}$ \\
\hline 5 & $\begin{array}{c}26,63 \\
a b\end{array}$ & $\begin{array}{c}31,81 \\
\mathrm{a}\end{array}$ & $\begin{array}{c}11,87 \\
\mathrm{a}\end{array}$ & $\begin{array}{c}57,08 \\
\mathrm{a}\end{array}$ & $\begin{array}{c}34,37 \\
\mathrm{a}\end{array}$ & $\begin{array}{c}91,44 \\
\mathrm{a}\end{array}$ & $8,25 \mathrm{a}$ & $5,35 \mathrm{a}$ & $\begin{array}{c}13,60 \\
\mathrm{a}\end{array}$ & $\begin{array}{c}77,85 \\
\mathrm{a}\end{array}$ & 3,50 & $\begin{array}{c}23,88 \\
\mathrm{a}\end{array}$ & $\begin{array}{c}87,30 \\
a\end{array}$ & $\begin{array}{c}4,43 \\
\mathrm{a}\end{array}$ \\
\hline \multicolumn{15}{|l|}{ Cultivar } \\
\hline Red & $\begin{array}{c}28,10 \\
\text { A }\end{array}$ & $\begin{array}{c}28,36 \\
\text { B }\end{array}$ & $\begin{array}{c}9,99 \\
\text { B }\end{array}$ & $\begin{array}{c}48,10 \\
\mathrm{~A}\end{array}$ & $\begin{array}{c}33,47 \\
\text { A }\end{array}$ & $\begin{array}{c}81,58 \\
\text { A }\end{array}$ & $\begin{array}{c}6,80 \\
\text { B }\end{array}$ & $\begin{array}{c}4,96 \\
\text { A }\end{array}$ & $\begin{array}{c}11,76 \\
\mathrm{~A}\end{array}$ & $\begin{array}{c}69,82 \\
\mathrm{~A}\end{array}$ & $\begin{array}{c}4,70 \\
\mathrm{~A}\end{array}$ & 21,85 & $\begin{array}{c}75,30 \\
\text { B }\end{array}$ & $\begin{array}{c}3,72 \\
\text { A }\end{array}$ \\
\hline Cherry & $\begin{array}{c}27,65 \\
\text { A }\end{array}$ & $\begin{array}{c}30,23 \\
\mathrm{~A}\end{array}$ & $\begin{array}{c}11,08 \\
\mathrm{~A}\end{array}$ & $\begin{array}{c}50,36 \\
\text { A }\end{array}$ & $\begin{array}{c}31,33 \\
\mathrm{~A}\end{array}$ & $\begin{array}{c}81,69 \\
\text { A }\end{array}$ & $\begin{array}{c}8,17 \\
\mathrm{~A}\end{array}$ & $\begin{array}{c}5,19 \\
\mathrm{~A}\end{array}$ & $\begin{array}{c}13,37 \\
\mathrm{~A}\end{array}$ & $\begin{array}{c}68,32 \\
\text { A }\end{array}$ & $\begin{array}{c}3,15 \\
\text { B }\end{array}$ & 20,65 & $\begin{array}{c}85,33 \\
\text { A }\end{array}$ & $\begin{array}{c}4,56 \\
\mathrm{~A}\end{array}$ \\
\hline S & $* *$ & $* *$ & $* *$ & $* *$ & NS & $* *$ & $* *$ & NS & $* *$ & $* *$ & NS & $* *$ & NS & $* *$ \\
\hline $\mathrm{C}$ & NS & $* *$ & $*$ & NS & NS & NS & $* *$ & NS & $* *$ & NS & $* *$ & NS & $*$ & $* *$ \\
\hline $\mathrm{S}^{*} \mathrm{C}$ & NS & NS & NS & NS & NS & $*$ & NS & NS & $*$ & NS & NS & NS & NS & $*$ \\
\hline $\mathrm{CV}(\%)$ & 20,76 & 6,92 & 13,07 & 14,65 & 24,18 & 7,15 & 14,84 & 24,07 & 7,05 & 7,50 & 41,68 & 18,47 & 15,96 & 9,57 \\
\hline
\end{tabular}

Substratos: $1=40 \%$ terra vermelha (Latossolo Vermelho Escuro), $40 \%$ casca pinus, $10 \%$ composição $1\left(\mathrm{C}_{1}\right)(40 \%$ casca de pinus $+30 \%$ vermiculita $+30 \%$ casca de arroz carbonizada), $10 \%$ composição $2\left(\mathrm{C}_{2}\right)(75 \%$ casca de pinus $+25 \%$ acícula de pinus $) ; 2=20 \%$ terra vermelha, $30 \%$ casca de pinus, $30 \% \mathrm{C}_{1}, 20 \% \mathrm{C}_{2} ; 3=50 \%$ casca de pinus, $20 \%$ fibra de coco granulada, $30 \% \mathrm{C}_{1} ; 4=30 \%$ casca de pinus, $30 \% \mathrm{C}$, $20 \%$ fibra de coco granulada, $20 \%$ fibra de coco mista; e $5=$ substrato comercial $(70 \%$ casca de pinus $+15 \%$ turfa $+15 \%$ vermiculita $)$.

Médias seguidas pela mesma letra não diferem entre si pelo teste Tukey a 5\%, sendo minúscula entre substratos e maiúscula entre cultivares. S: substrato. C: cultivar. NS: não significativo; *** significativo a 1 e $5 \%$, respectivamente.

Means followed by the same lowercase letters in the same column and uppercase letters in the same line did not differ by the Tukey test at $5 \%$. C: cultivar, NS: not significant; ** significat at 1\%, * significant at 5\%. 
Tabela 4. Valores médios de fitomassa fresca e seca total e diâmetro dA haste em plantas de gérbera de vaso. Botucatu (SP). 2008

Table 4. Mean values of fresh and dry stem diameter and total plant gérbera pot. Botucatu (SP). 2008

\begin{tabular}{|c|c|c|c|c|c|c|}
\hline \multirow{3}{*}{ Substrato } & \multicolumn{2}{|c|}{ Fitomassa fresca total } & \multicolumn{2}{|c|}{ Fitomassa seca total } & \multicolumn{2}{|c|}{ Diâmetro de haste } \\
\hline & Red & Cherry & Red & Cherry & Red & Cherry \\
\hline & \multicolumn{4}{|c|}{--------------------------------g------------------------------- } & \multicolumn{2}{|c|}{----------mm---------- } \\
\hline 1 & $68,31 \mathrm{~b} \mathrm{~A}$ & $71,78 \mathrm{~b} \mathrm{~A}$ & $10,11 \mathrm{~b} \mathrm{~B}$ & $12,10 \mathrm{~cd} \mathrm{~A}$ & $3,08 \mathrm{c} \mathrm{B}$ & 4,28 a A \\
\hline 2 & $51,24 \mathrm{c} \mathrm{B}$ & $61,40 \mathrm{~b} \mathrm{~A}$ & $7,51 \mathrm{c} \mathrm{B}$ & $10,89 \mathrm{~d} A$ & $3,17 \mathrm{bc} \mathrm{B}$ & 4,72 a $\mathrm{A}$ \\
\hline 3 & 92,61 a A & 91,94 a A & 13,15 a $\mathrm{A}$ & $14,25 \mathrm{ab} A$ & $3,98 \mathrm{ab} \mathrm{A}$ & 4,42 a $\mathrm{A}$ \\
\hline 4 & 100,89 a $\mathrm{A}$ & 95,25 a A & 14,70 a A & 15,73 a A & 4,04 a B & 4,85 a $\mathrm{A}$ \\
\hline 5 & 92,62 a A & 88,06 a $\mathrm{A}$ & 13,34 a A & 13,86 bc A & 4,32 a $\mathrm{A}$ & 4,54 a A \\
\hline
\end{tabular}

Substratos: $1=40 \%$ terra vermelha (Latossolo Vermelho Escuro), $40 \%$ casca pinus, $10 \%$ composição $1\left(\mathrm{C}_{1}\right)(40 \%$ casca de pinus $+30 \%$ vermiculita + $30 \%$ casca de arroz carbonizada), $10 \%$ composição $2\left(\mathrm{C}_{2}\right)(75 \%$ casca de pinus $+25 \%$ acícula de pinus $) ; 2=20 \%$ terra vermelha, $30 \%$ casca de pinus, $30 \% \mathrm{C}_{1}, 20 \% \mathrm{C}_{2} ; 3=50 \%$ casca de pinus, $20 \%$ fibra de coco granulada, $30 \% \mathrm{C}_{1} ; 4=30 \%$ casca de pinus, $30 \% \mathrm{C}_{1}, 20 \%$ fibra de coco granulada, $20 \%$ fibra de coco mista; e $5=$ substrato comercial ( $70 \%$ casca de pinus $+15 \%$ turfa $+15 \%$ vermiculita).

Médias seguidas pela mesma letra minúscula na coluna e maiúscula na linha, não diferem entre si pelo teste Tukey a $5 \%$.

Means followed by the same lowercase letters in the same column and uppercase letters in the same line did not differ by the Tukey test at $5 \%$.

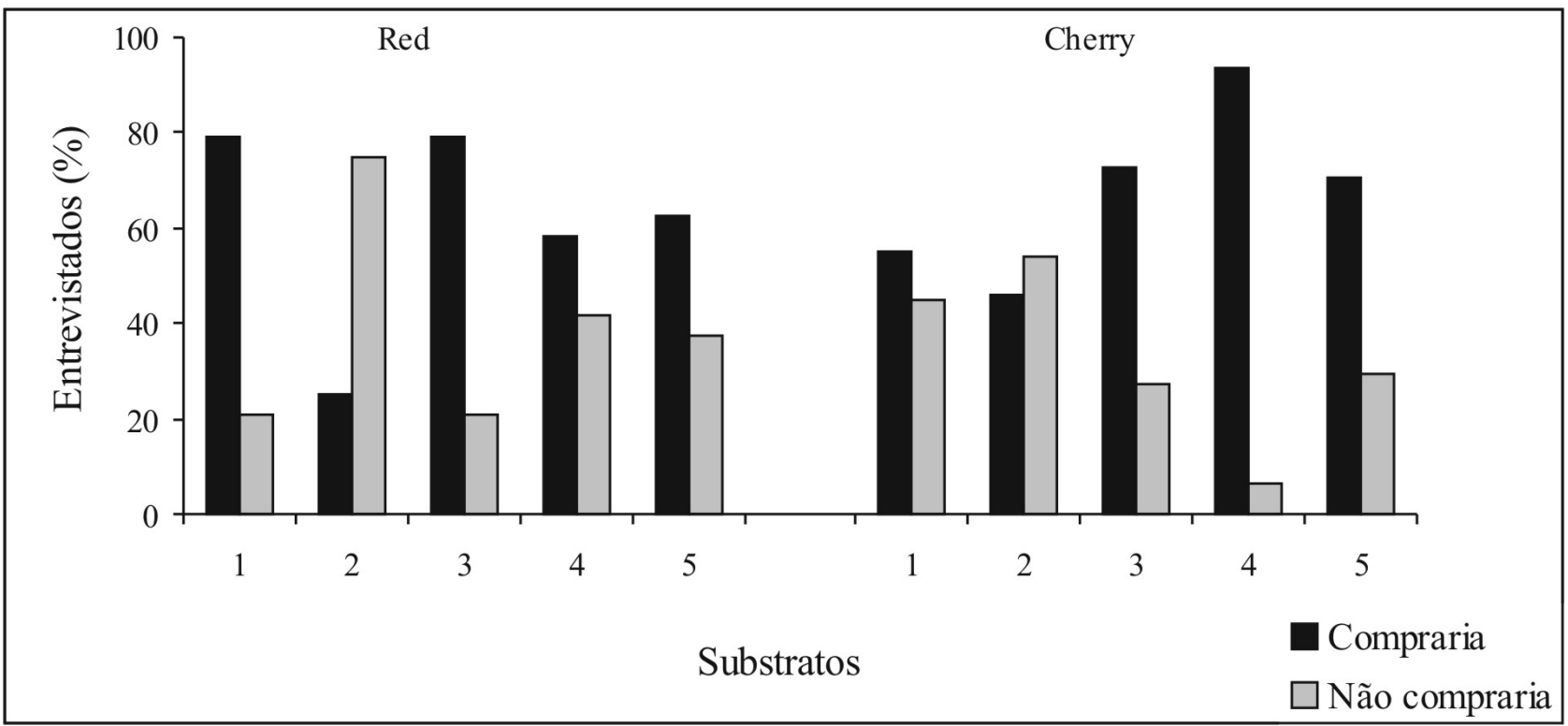

Substratos: 1- 40\% Latossolo Vermelho Escuro (LVE) $+40 \%$ casca de pinus $(\mathrm{CP})+10 \%$ composição $1\left(\mathrm{C}_{1}\right)(40 \% \mathrm{CP}+30 \%$ vermiculita $(\mathrm{V})+30 \%$ casca de arroz carbonizada) $+10 \%$ composição $2\left(\mathrm{C}_{2}\right)(75 \% \mathrm{CP}+25 \%$ acícula de pinus $), 2-20 \% \mathrm{LVE}+30 \% \mathrm{CP}+30 \% \mathrm{C}+20 \% \mathrm{C}, 3-50 \% \mathrm{CP}+$ $20 \%$ fibra de coco granulada $(\mathrm{FCG})+30 \% \mathrm{C}_{1}, 4-30 \% \mathrm{CP}+30 \% \mathrm{C}_{1}+20 \% \mathrm{FCG}+20 \%$ fibra de coco mista, $5-$ substrato comercial $(70 \% \mathrm{CP}+15 \%$ turfa $+15 \% \mathrm{~V})$

Figura 1. Reação dos entrevistados quando questionados sobre a compra das cultivares Red e Cherry produzidas nos diferentes substratos. Botucatu (SP). 2008.

Figure 1. Reaction of interviewed when questioned about the acquisition of Red and Cherry cultivars grown in different substrates. Botucatu (SP). 2008. 